\title{
In situ synchrotron X-ray diffraction study of deformation behaviour of a metastable $\beta$-type Ti-33Nb-4Sn alloy
}

\author{
Shun Guo ${ }^{\mathrm{a}, \mathrm{b}, \mathrm{d}}$, Yao Shang ${ }^{\mathrm{a}}$, Junsong Zhang ${ }^{\mathrm{a}, *}$, Qingkun Meng ${ }^{\mathrm{b}, \mathrm{c}}$, Xiaonong Cheng ${ }^{\mathrm{a}}$, \\ Xinqing Zhao ${ }^{\mathrm{b}, *}$
}

\begin{abstract}
In this study, the deformation behaviour of metastable $\beta$-type Ti-33Nb-4Sn alloys in different thermo-mechanical treatment states is investigated by tensile tests and in situ synchrotron X-ray diffraction (SXRD). In the case of solution-treated alloy, stress-induced martensitic (SIM) transformation takes place over a wide strain range of $0.5-14 \%$. During this SIM transformation, the parameter of $b_{\alpha^{\prime \prime}}\left([020]_{\alpha^{\prime \prime}}\right)$ increases with macroscopic strain within strain range of $1.5-4.7 \%$, giving rise to that the $\alpha^{\prime \prime}$ variants, with $b_{\alpha^{\prime \prime}}$-axis $\left([020]_{\alpha^{\prime \prime}}\right)$ parallel to tensile direction, are formed preferentially to accommodate the macroscopic strain during loading. Similar SIM transformation behaviour also occurs in cold-rolled alloy with the exception that the extent of SIM
\end{abstract}


transformation and pre-existing $\alpha^{\prime \prime}$ variants reorientation is much slighter than that in solution-treated specimen. This slighter SIM transformation gives rise to nonlinear deformation, instead of "stress plateau", in the cold-rolled alloy. Upon annealing, the $\beta$ phase survives against SIM transformation due to the suppression effects of dislocations and grain boundaries, resulting in a huge elastic deformability. Based on the results of tensile tests and SXRD, the activation sequence of different deformation mechanisms and the regions of different deformation mechanisms of Ti-33Nb-4Sn alloys, in solution-treated, cold-rolled and annealed states, are clarified unambiguously.

Keywords : Titanium alloys; Tensile deformation behaviour; Phase transformation; Huge elastic deformability.

\section{Introduction}

Titanium and its alloys are generally considered to be the most suitable biomaterials for orthopedic implants due to their light weight, high corrosion resistance, excellent biocompatibility, good mechanical properties including relatively low elastic modulus [1-3]. Currently, the most widely used Ti material for implants, $(\alpha+\beta)$ type Ti-6Al-4V, has an elastic modulus of about $110 \mathrm{GPa}$, which cannot match well with that of natural human bone (about $30 \mathrm{GPa}$ ) and hence results in "stress shielding" and eventual implant failure [4,5]. Additionally, it has been reported that some long-term health problems, e.g. Alzheimer disease and neuropath, might be induced by the toxic $\mathrm{V}$ and $\mathrm{Al}$ ions released from Ti-6Al-4V [4,6]. Consequently, more and more efforts have been made to develop non-cytotoxic metastable $\beta$-type Ti alloys with lower elastic modulus than Ti-6Al-4V alloy [7-10].

Previous studies have shown that the elastic moduli of metastable $\beta$-type $\mathrm{Ti}$ 
alloys are mainly determined by their $\beta$-phase stability, and that the elastic modulus increases with increasing the stability of $\beta$-phase in most metastable $\beta$-type Ti alloys [10-12]. Meanwhile, the increase in $\beta$-phase stability can lead to an obvious change in the deformation behaviour of $\beta$-phase Ti alloys, involving mainly stress-induced martensitic (SIM) transformation from $\beta$ phase (bcc, space group $\operatorname{Im} 3 m$ ) to $\alpha$ " martensite (C-orthorhombic, space group $\mathrm{Cmcm}$ ), twinning and plastic slip [13-17]. Among these deformation behaviour, SIM transformation is of special concern, because this SIM transformation gives rise to shape memory effect and superelasticity in metastable $\beta$-type Ti alloys which are being considered as a potential candidate to replace toxic NiTi-based shape memory alloys in biomedical applications $[18,19]$. By adjusting $\beta$-phase stability, the metastable $\beta$-type Ti alloys can exhibit single $\alpha^{\prime \prime}$ martensite or single $\beta$ phase upon quenching from high temperature $\beta$-phase field. The former is expected to possess shape memory effect, while the latter is expected to exhibit superelasticity [20,21]. Logically, a mixture of $\beta$ and $\alpha^{\prime \prime}$ phases is obtained when the stability of $\beta$-phase locates between the above two cases. In this case, neither excellent shape memory effect nor perfect superelasticity is obtained in the metastable $\beta$-type $\mathrm{Ti}$ alloys due to an incomplete martensitic transformation. Therefore, little attention was paid to investigate the SIM transformation behaviour in metastable $\beta$-type Ti alloys containing a mixture of $\beta$ and $\alpha^{\prime \prime}$ phases, although numerous investigations have concentrated on the SIM transformation behaviour in single $\alpha^{\prime \prime}$ or $\beta$-phase Ti alloys [22-25].

Recently, it was found that metastable $\beta$-type Ti alloys, which exhibit a mixture of $\beta$ and $\alpha^{\prime \prime}$ phases upon quenching, have a high potential for orthopedic implants owing to their ultralow elastic modulus [26-29]. Typically, Ti-33Nb-4Sn (wt. \%) alloy, a composition showing a dual $\beta$ and $\alpha$ " phases upon quenching, can exhibit an 
ultralow Young's modulus of $36 \mathrm{GPa}$ after appropriate cold rolling plus annealing [29]. Further investigation shows that this ultralow modulus is closely associated with its low $\beta$-phase stability with respect to $\alpha^{\prime \prime}$ martensitic transformation [30]. It is also shown that the Ti-33Nb-4Sn alloy exhibits multiple deformation behavior, involving “double yielding", nonlinear deformation and conventional linear elastic deformation, under different thermo-mechanical treatment conditions [29,30]. However, the deformation behaviour of the Ti-33Nb-4Sn alloy, especially its correlation with SIM transformation, has not yet been investigated detailedly, although an in-depth understanding of this issue might help to establish theoretical foundations for designing novel biomedical Ti alloys with ultralow elastic modulus.

Laboratory X-ray diffraction may appear to provide a solution for studies of deformation behaviour of the metastable $\beta$-type $\mathrm{Ti}$ alloys, especially that related to SIM transformation [31]. However, the low sensitivity of conventional X-ray sources, caused by long wavelength and the coexistence of both $\mathrm{K}_{\alpha 1}$ and $\mathrm{K}_{\alpha 2}$ components [32], is not always sufficient to give an accurate depiction of what is actually taking place in alloys. In comparison to conventional X-ray sources, in situ synchrotron X-ray source can provide a higher level of detail due to its shorter wavelength and better monochromaticity. Furthermore, in situ synchrotron X-ray source allows the SIM transformation of a common $\{h \mathrm{hl}\}$ plane in a specific direction to be studied individually, which is impossible for conventional X-ray sources because the latter can only provide average phase transformation information of the surface of specimen.

This article presents in situ synchrotron X-ray diffraction observations of a recently developed Ti-33Nb-4Sn (wt. \%) alloy during uniaxial tensile. Special attention is focused on the relationship between SIM transformation and deformation 
behaviour in this alloy. On the basis of the characterization results of crystal structure, phase fraction, lattice parameters, etc., the deformation mechanisms of metastable $\beta$-type Ti-33Nb-4Sn alloys under different thermo-mechanical treatment conditions were discussed.

\section{Experimental procedure}

The material used in the present study has a chemical composition of Ti-33Nb-4Sn (wt. \%). After melting, homogenizing, forging, solution-treating (at $1073 \mathrm{~K}$ for 1 hour $)$ and quenching into water $(298 \mathrm{~K})$, the solution-treated ingot was fabricated. The ingot was cold rolled into a plate with a thickness of $\sim 1 \mathrm{~mm}$, by a thickness reduction of $\sim 87 \%$. The detail preparation process of Ti-33Nb-4Sn alloy can be seen in a previous reference [29]. In this study, the specimens that were electric discharge machined from the cold-rolled plate are abbreviated as CR specimens. Part of $\mathrm{CR}$ specimens was again solution-treated at $1073 \mathrm{~K}$ for 1 hour, followed by quenching into water (i.e., equivalent to the solution-treated state before cold rolling). This type of specimens is referred to as ST specimen henceforth. Part of CR specimens was annealed at $673 \mathrm{~K}$ for 20 minutes, followed by quenching into water. This type of specimens is abbreviated as CRA specimen henceforth.

Conventional X-ray diffraction (XRD) characterization was conducted on a Rigaku D/max 2550 diffractometer with $\mathrm{Cu} \mathrm{K \alpha}$ source $(\lambda=1.5418 \AA$ ) operating at an accelerating voltage of $40 \mathrm{kV}$ and a current of $200 \mathrm{~mA}$. Microstructural observations were carried out on a FEI Quanta 200F transmission electron microscope (TEM) operating at a voltage of $200 \mathrm{kV}$. Thin foils for TEM were prepared by the twin-jet electropolishing technique using a solution of $9 \%$ perchloric acid, $21 \%$ n-butyl alcohol and balance methanol. Macroscopic uniaxial tensile test was performed on an Instron-8801 testing system at a strain rate of $1 \times 10^{-3} \mathrm{~s}$. Tensile specimens were 
electric discharge machined along the original cold rolling direction into the following shapes: 1-mm thickness, 1.46-mm width, and 30-mm gage length. In situ synchrotron X-ray diffraction (SXRD) data were collected on beam line 11-ID-C at the Advanced Photon Source (APS) at Argonne National Laboratory. A monochromatic X-ray beam with a wavelength of $0.10798 \AA$ (with an energy of $115 \mathrm{keV}$ ) and beam size of 0.6 $\mathrm{mm} \times 0.6 \mathrm{~mm}$ was employed to capture two-dimensional (2-D) Debye-Scherrer diffraction patterns during uniaxial tensile loading, and a schematic set-up of the in situ synchrotron X-ray diffraction measurement is shown in Fig. S1 (see supplementary information). The cylindrical specimen was first mechanically ground and polished, then mounted on a tensile loading rig. During the whole SXRD test, the specimens were stretched in the original rolling direction, and the samples were positioned such that the diffraction data originating from crystal grains with the plane normal aligned to the loading direction were collected at the $90^{\circ}$ and $270^{\circ}$ azimuthal angles of the Debye-Scherrer rings. The Debye-Scherrer rings were calibrated, using Fit2d software and a standard $\mathrm{CeO}_{2}$ calibration sample to refine the beam center, the sample-detector distance and detector misalignment, and output one-dimensional (1-D) patterns for further analysis.

\section{Results and discussion}

\subsection{Deformation behaviour of ST Ti-33Nb-4Sn alloy}

After quenching from high temperature $\beta$-phase field, lath-shaped $\alpha$ " martensite is formed within the coarse $\beta$ parent phase. More details on microstructure can be found in a previous work [29]. Here, the initial SXRD 1-D pattern of ST Ti-33Nb-4Sn alloy before loading is shown in Fig. 1a. A mixture of $\beta$ parent phase and $\alpha^{\prime \prime}$ martensite is formed in $\mathrm{ST}$ Ti-33Nb-4Sn alloy, visible as the diffraction peaks from both $\beta$ and $\alpha^{\prime \prime}$ phases, such as $\{110\}_{\beta},(002)_{\alpha^{\prime \prime}}$ and $(020)_{\alpha^{\prime \prime}}$. Some weak diffraction 
peaks from $\alpha^{\prime \prime}$ martensite, e.g. $(221)_{\alpha^{\prime \prime}},(202)_{\alpha^{\prime \prime}},(220)_{\alpha^{\prime \prime}}$ and $(131)_{\alpha^{\prime \prime}}$, can be seen more clearly in the inset of Fig. 1a. This suggests that the martensitic transformation start (Ms) temperature of ST Ti-33Nb-4Sn alloy is higher than room temperature, which is reasonably attributed to the low total amount of $\beta$ stabilizers in the present alloy. Fig. $1 \mathrm{~b}$ gives the convention XRD pattern of $\mathrm{ST}$ Ti-33Nb-4Sn alloy within the 2theta range of $37-40^{\circ}$. No visible diffraction peak from $(002)_{\alpha^{\prime \prime}}$ is detected in this figure, while this $(002)_{\alpha^{\prime \prime}}$ peak can be detected clearly alongside $\{110\}_{\beta}$ by SXRD technique (Fig. 1a). This implies that in comparison to conventional XRD, SXRD has higher resolution and thus can provide a higher level of detail in phase transformation.

Fig. 2a gives part of the straightened Debye-Scherrer diffraction rings of ST specimen as a function of macroscopic strain. Generally speaking, the intensity of $\beta$ spots decreases with increasing macroscopic strain, together with an increase in the intensity of $\alpha^{\prime \prime}$ martensite, implying that a progressive stress-induced martensitic (SIM) transformation takes place during deformation. Additionally, the distribution of diffraction intensity of $\beta$ and $\alpha^{\prime \prime}$ spots along the straightened diffraction rings becomes more and more nonuniform with increasing macroscopic strain, suggesting that the texture of both $\beta$ and $\alpha^{\prime \prime}$ phases are intensified by the SIM transformation during deformation. The highly developed texture can provide visualized information on the crystallographic orientation relationship between the parent $\beta$ and $\alpha^{\prime \prime}$ phases. Fig. $2 b$ shows the original 2-D Debye-Scherrer diffraction rings of ST specimen acquired at a strain of $14 \%$, where the diffraction rings arising from $\beta$ and $\alpha^{\prime \prime}$ are indexed, respectively. In Fig. $2 b$, it can be seen that the martensitic transformation between $\beta$ and $\alpha$ " obeys the following crystallographic orientation relationship: $(100)_{\beta} / /(100)_{\alpha " \text {, }}$ $(011)_{\beta} / /(010)_{\alpha^{\prime \prime}}$ and $(0 \overline{1})_{\beta} / /(001)_{\alpha^{\prime \prime}}$. Additionally, the angles between some $\beta$ and $\alpha^{\prime \prime}$ crystal planes can also be determined directly, e.g., $54.6 \pm 1^{\circ}$ for the angle between the 
$(011)_{\beta}$ and $(110)_{\alpha^{\prime \prime}}$ planes and $32.2 \pm 1^{\circ}$ for the angle between $(011)_{\beta}$ and $(131)_{\alpha^{\prime \prime}}$, respectively. These results agree well with the calculative results of the crystallographic orientation relationship between $\beta$ and $\alpha^{\prime \prime}$ phases using phenomenological theory in a previous literature [33].

In order to characterize more precisely the SIM transformation of ST Ti-33Nb-4Sn alloy, the evolution of SXRD 1-D patterns, relative integrated intensity and parameters of $\beta$ and $\alpha "$ phases as a function of macroscopic strain is concerned. Fig. 3 displays the evolution of SXRD 1-D patterns (within the $d$-spacing range of 2.2-2.6 $\AA$ ) with macroscopic strain in the loading direction (i.e., a $10^{\circ}$ azimuthal bin around $90^{\circ}$ and $270^{\circ}$, Fig. 3a) and in the Poisson direction (i.e., a $10^{\circ}$ azimuthal bin around $0^{\circ}$ and $180^{\circ}$, Fig. 3b), respectively. From Fig. 3(a), it is seen that the $d$-spacing of $\{110\}_{\beta}$ diffraction peak (labeled by the blue circle) increases monotonically with macroscopic strain up to a strain of $\sim 4.7 \%$, where the $\{110\}_{\beta}$ peak disappears in the loading direction. In contrast, the $(002)_{\alpha^{\prime \prime}}$ peak (labeled by the black diamond) decreases with increasing macroscopic strain and finally disappears at a strain of $\sim 2.1 \%$. Referring to $(020)_{\alpha^{\prime \prime}}$ diffraction peak (labeled by the red square), it increases firstly with increasing macroscopic strain to $\sim 4.7 \%$ and then remains almost constant with a further increase in strain in the loading direction. Additionally, the $(020)_{\alpha "}$ diffraction peak intensifies with macroscopic strain in the loading direction (Fig. 3a), accompanied by the decrease and even disappearance of $(020)_{\alpha^{\prime \prime}}$ in the Poisson direction (inset of Fig. 3b). This is attributed to the combined effects of SIM transformation and reorientation of martensite variants, which will be discussed in detail later. From Fig. 3b, the $\{110\}_{\beta}$ diffraction peak can be observed clearly during the whole loading in the Poisson direction, even at a high macroscopic strain of $14 \%$, which is obviously different from the observation that the $\{110\}_{\beta}$ peak disappears at a 
macroscopic strain of $\sim 4.7 \%$ in the loading direction (Fig. 3a). This is in good accordance with the previously published results that SIM transformation and reorientation of martensite variants are strongly dependent on the crystallographic orientation with respect to the external stress [34]. Thus, in the present study, the volume fractions of the $\beta$ and $\alpha^{\prime \prime}$ phases were characterized by the integrated intensity of the whole Debye-Scherrer diffraction rings (i.e., $0^{\circ} \leq$ azimuthal angles $\leq 360^{\circ}$ ), since the intensity of diffraction peaks in a certain direction, such as the intensity of $\{110\}_{\beta}$ peak in the loading direction, is inadequate to precisely characterize the volume fractions of $\beta$ and $\alpha^{\prime \prime}$ phases as a whole.

Fig. 4 represents the evolution of the relative integrated intensity of $\{110\}_{\beta}$, $(002)_{\alpha^{\prime \prime}}$ and (020) $)_{\alpha^{\prime \prime}}$ peaks (Fig. 4a), and the parameters of $\beta$ (Fig. 4b) and $\alpha^{\prime \prime}$ (Fig. 4c) phases of ST Ti-33Nb-4Sn specimen during loading. It can be seen from Fig. 4a that the relative integrated intensity of $\{110\}_{\beta}$ deceases drastically at a high rate within the strain range of $0.5 \sim 4.7 \%$, and then decreases slightly at a low rate up to a strain of $\sim 14 \%$. Meanwhile, the relative integrated intensity of $(002)_{\alpha^{\prime \prime}}$ and $(020)_{\alpha^{\prime \prime}}$ starts to increase at $\sim 0.5 \%$ strain, and increases significantly to $4.7 \%$ strain, and then increases gradually to $14 \%$ strain. This indicates that the SIM transformation takes place intensively in the strain range from $0.5 \%$ to $4.7 \%$ but slightly from $4.7 \%$ to $14 \%$. Note that the SIM transformation in the ST Ti-33Nb-4Sn alloy is incomplete, with some residual $\beta$ not being transformed, as evidenced by the fact that a relative integrated intensity of 0.79 for $\{110\}_{\beta}$ peak is still observed at $14 \%$ strain. Additionally, it should also be noted that the whole integrated intensity of $(002)_{\alpha^{\prime \prime}}$ shows an increasing trend from $\sim 0.5 \%$ strain, though the $(002)_{\alpha^{\prime \prime}}$ diffraction peak decreases with increasing strain from $\sim 0.5 \%$ strain and disappears at $\sim 2.1 \%$ strain in the loading direction (Fig. 3a). This suggests that besides SIM transformation, the reorientation of pre-existing 
martensite variants (i.e., that were formed upon quenching) takes place within a macroscopic strain of $0.5-2.1 \%$.

From Fig. 4b, it is seen that the parameter of $a_{\beta}$ first increases with macroscopic strain to $4.7 \%$ and then stays about constant with further increase of macroscopic strain. Clearly, the increase in $a_{\beta}$ is due to the elastic deformation of $\beta$ phase, while the constant of $a_{\beta}$, regardless of further increase in strain, is ascribed to the plastic deformation of residual $\beta$. Referring to the three lattice parameters $\left(a_{\alpha^{\prime \prime}}, b_{\alpha^{\prime \prime}}\right.$ and $\left.c_{\alpha^{\prime \prime}}\right)$ of $\alpha^{\prime \prime}$ phase shown in Fig. 4c, one can see that the $a_{\alpha \text { " }}$ keeps approximately constant during the entire tensile loading, while the parameter of $c_{\alpha^{\prime \prime}}$ stays roughly constant firstly, then deceases slightly in the strain range of $1.5-4.7 \%$, and finally keeps approximately constant with further loading. In comparison to $\mathrm{a}_{\alpha \text { " }}$ and $\mathrm{c}_{\alpha " \text {, }}$, the evolution of $b_{\alpha^{\prime \prime}}$ is more valuable, although seemingly more complex. The $b_{\alpha^{\prime \prime}}$ first increases slightly with increasing strain to $0.5 \%$, which corresponds to the elastic deformation of pre-existing $\alpha^{\prime \prime}$ phase that was formed during quenching. Then, the parameter of $b_{\alpha^{\prime \prime}}$ stays roughly constant in the macroscopic strain range of $0.5-1.5 \%$. When the macroscopic strain locates between $1.5 \%$ and $4.7 \%$, the $b_{\alpha \prime}$ increases monotonically with macroscopic strain, corresponding to the elastic deformation of the re-oriented and stress-induced $\alpha^{\prime \prime}$ phase. With further increasing strain to $14 \%$, the

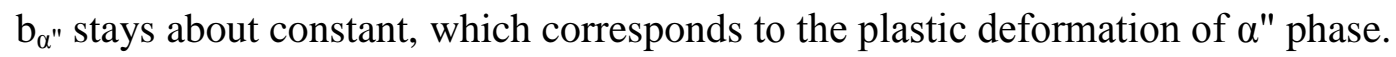

On the basis of the above evolution of the lattice parameters of $\beta$ and $\alpha$ ", we can offer a reasonable explanation for the reason why the combined influence of SIM transformation and martensite variants reorientation gives rise to strengthening $(020)_{\alpha "}$ peak, but reducing and even eliminating $(002)_{\alpha^{\prime \prime}}$ and $\{110\}_{\beta}$ in the loading direction (Fig. 3a). Based on the orientation relationship between $\beta$ phase and $\alpha$ " martensite, part of $\langle 100\rangle_{\beta}$ are converted into $[100]_{\alpha^{\prime \prime}}$ (i.e., $a_{\alpha "}$-axis) and part of $\langle 110\rangle_{\beta}$ are 
changed into $[020]_{\alpha^{\prime \prime}}\left(\right.$ i.e., $b_{\alpha^{\prime \prime}}$-axis) or $[002]_{\alpha^{\prime \prime}}$ (i.e., $c_{\alpha^{\prime \prime}}$-axis), resulting in 6 equivalent lattice correspondences between the $\beta$ and $\alpha^{\prime \prime}$ phases and the corresponding 12 martensite variants $[35,36]$. In the case of quenching from $\beta$-phase region, various martensite variants are formed in a self-accommodation manner [23], which is proven by the coexistence of many kinds of $\alpha^{\prime \prime}$ martensite peaks in a certain orientation, such as the coexistence of $(020)_{\alpha^{\prime \prime}}$ and (002) $)_{\alpha^{\prime \prime}}$ in the loading direction (Fig. 1a). But, when the $\alpha^{\prime \prime}$ martensite formed during quenching is subjected to the external stress or a SIM transformation occurs due to the presence of the applied stress, the reorientation of pre-existing martensite variants or the selection of brand-new martensite variants will be activated, giving rise to that only the variants which can provide a maximum strain, i.e., accommodate the macroscopic strain to the greatest extent, will survive or be formed during loading [34,37]. In the present Ti-33Nb-4Sn alloy, the $b_{\alpha \text { " }}$ increases with macroscopic strain in the strain range of $1.5-4.7 \%$, whereas the $a_{\alpha "}$ and $c_{\alpha^{\prime \prime}}$ stay about constant or reduce with macroscopic strain, respectively (Fig. 4c). Thus, it is expected that in the case of Ti-33Nb-4Sn alloy, the $\alpha^{\prime \prime}$ variants, with $b_{\alpha^{\prime \prime}}$-axis $\left([020]_{\alpha^{\prime \prime}}\right)$ being aligned to tensile direction and $\mathrm{a}_{\alpha^{\prime \prime}}$ and $\mathrm{c}_{\alpha^{\prime \prime}}$ axis $\left([100]_{\alpha^{\prime \prime}}\right.$ and $\left.[002]_{\alpha^{\prime \prime}}\right)$ being perpendicular to tensile direction, will be formed favorably to accommodate the macroscopic strain in tensile loading. Indeed, this is in accordance with the observation that during loading, the $(020)_{\alpha^{\prime \prime}}$ diffraction peak increases but the $(002)_{\alpha "}$ decreases with macroscopic strain in the loading direction, accompanied by the decrease and even disappearance of $(020)_{\alpha^{\prime \prime}}$ in the Poisson direction (Fig. 3).

According to the results shown above, the domains of different deformation mechanisms of ST Ti-33Nb-4Sn alloy are distinguished straightforwardly and depicted on the macroscopic stress-strain curve of ST Ti-33Nb-4Sn specimen in Fig. $4 \mathrm{~d}$. At strains below $0.5 \%$, the pre-existing $\alpha^{\prime \prime}$ martensite and $\beta$ phase simultaneously 
undergo elastic deformation. Then, the reorientation of pre-existing $\alpha$ " variants takes place within the strain range from $0.5 \%$ to $2.1 \%$, as evidenced by the fact that the $(020)_{\alpha "}$ diffraction peak intensifies with macroscopic strain in the loading direction (Fig. 3a), accompanied by the decrease and even disappearance of $(020)_{\alpha^{\prime \prime}}$ in the Poisson direction (inset of Fig. 3b). Meanwhile, the SIM transformation occurs simultaneously, as suggested by the fact that the relative integrated intensity of $\{110\}_{\beta}$ deceases but the relative integrated intensity of $(002)_{\alpha^{\prime \prime}}$ and $(020)_{\alpha^{\prime \prime}}$ increase within this strain range (Fig. 4a). In the macroscopic strain range covering $1.5-4.7 \%$, the re-oriented and stress-induced $\alpha$ " martensite is deformed elastically, accompanied by the elastic deformation of the remaining $\beta$ parent phase and the continuing SIM transformation. At higher strains above 4.7\%, the re-oriented and stress-induced $\alpha^{\prime \prime}$ phase and the remaining $\beta$ phase are primarily deformed plastically. At this stage, the SIM transformation between $\beta$ to $\alpha^{\prime \prime}$ phases still takes place at a relatively low rate, as shown in Fig. 4c. This can be mainly ascribed to the fact that when the strain is in excess of $4.7 \%$, the macroscopic stress does not considerably increase with macroscopic stain any longer, owing to the onset of plastic deformation.

\subsection{Deformation behaviour of CR Ti-33Nb-4Sn alloy}

Upon a severe cold rolling deformation, numerous dark regions originating from dislocation tangles are observed in CR Ti-33Nb-4Sn alloy, as shown in Fig. 5a. In contrast, no visible dislocation tangles are seen in ST Ti-33Nb-4Sn alloy (Fig. 5b), suggesting that the formation of dislocation tangles is attributed to severe plastic deformation caused by cold rolling. From the selected area electron diffraction (SAD) pattern of CR specimen (shown in the top right corner of Fig. 5a), one can see that after the cold rolling deformation, the Ti-33Nb-4Sn alloy still consists of $\beta$ phase and $\alpha$ " martensite, indicating that the present cold rolling does not change the phase 
constitutions of the Ti-33Nb-4Sn alloy. Nevertheless, this cold rolling gives rise to considerable grain refinement, as evidenced by the near-continuous diffraction rings that are apparently different from isolated diffraction spots for ST specimen (shown in the top right corner of Fig. 5b). This refinement effect of cold rolling is commonly observed in Ti-Nb based alloys, especially in metastable $\beta$-type Ti-Nb based alloys with excellent cold workability [38,39].

Fig. 6a represents part of the straightened Debye-Scherrer diffraction rings of CR specimen at different strain levels during uniaxial loading. It is seen that the intensity of $\alpha^{\prime \prime}$ spots intensifies with macroscopic strain, whereas at the same time the intensity of $\beta$ spots subsides with macroscopic strain, indicating that a progressive SIM takes place during uniaxial loading. This SIM transformation exacerbates the texture of both $\beta$ and $\alpha^{\prime \prime}$ phases, visible as more and more uneven distribution of diffraction intensity of $\beta$ and $\alpha^{\prime \prime}$ spots along the straightened diffraction rings, which is similar to that observed in ST Ti-33Nb-4Sn specimen. Additionally, similar to ST specimen, CR specimen also shows an incomplete SIM transformation, visible as some residual $\{110\}_{\beta}$ diffraction spots at a strain of $5 \%$.

Fig. 6b displays the evolution of SXRD 1-D patterns (within the $d$-spacing range of 2.2-2.6 ) with macroscopic strain for $\mathrm{CR}$ specimen in the loading direction. A comparison of the initial patterns (i.e., at a macroscopic strain of $0 \%$ ) between the CR and ST (Fig. 3a) specimens reveals that the initial intensity of $\{110\}_{\beta}$ diffraction peak in CR specimen is much lower than that in ST specimen. Obviously, this is attributed to the severe cold rolling deformation process during which a lot of residual $\beta$ phase surviving after quenching was transformed into $\alpha "$ martensite. From Fig. 6b, one can see that the diffraction intensity of $(020)_{\alpha "}$ peak (labeled by the red square) intensifies with macroscopic strain. Meanwhile, the diffraction intensity of $\{110\}_{\beta}$ peak (marked 
by the blue circle) and (002) $)_{\alpha "}$ peak (marked by the black diamond) reduces monotonically with macroscopic strain up to a strain of $\sim 3.5 \%$, where both $\{110\}_{\beta}$ and $(002)_{\alpha^{\prime \prime}}$ peaks almost vanish. The disappearance of $\{110\}_{\beta}$ and $(002)_{\alpha^{\prime \prime}}$ peaks is ascribed to the combined effect of SIM transformation and reorientation of pre-existing $\alpha^{\prime \prime}$ martensite, which is similar to that occurred in ST specimen.

But, the extent of SIM transformation is quite different in ST and CR specimens. Fig. 7a shows the evolution of the relative integrated intensity as a function of macroscopic strain during loading. It can be observed that the relative integrated intensity of $\{110\}_{\beta}$ peak first reduces with macroscopic strain up to $\sim 3.5 \%$ and then remains almost constant with further increasing strain to $\sim 5 \%$. Meanwhile, the relative integrated intensity of $(002)_{\alpha "}$ peak and $(020)_{\alpha^{\prime \prime}}$ peak increases with macroscopic strain to $\sim 3.5 \%$ and then stays about constant with further increasing strain to $5 \%$, indicating that a progressive SIM takes place within the strain range of $0-3.5 \%$ during the uniaxial loading. A comparative analysis of Fig. 4a and Fig. 7a shows that the reduction in the relative integrated intensity of $\{110\}_{\beta}$ during the whole loading is only $\sim 0.07$ for CR specimen, much lower than a value of $\sim 0.20$ for ST specimen. This suggests that the extent of SIM transformation in CR specimen is much smaller than that in ST specimen. The difference in the extent of SIM transformation between ST and CR specimens can be rationalized from the following two important facts: I ) before tensile loading (i.e., at a macroscopic strain of 0\%), the volume fraction of $\beta$ in CR specimen is much lower than that in ST specimen due to the SIM transformation occurred during cold rolling, as shown in Fig. 3 and Fig. 6b; II ) As is well known, martensitic transformation can be suppressed effectively by some structural factors, such as grain refinement and dislocation tangles, due to the shear nature of martensitic transformation $[26,40,41]$. In the case of $\mathrm{CR}$ Ti-33Nb-4Sn 
specimen, a lot of dislocations and grain boundaries caused by cold rolling (Fig. 5a) play a key role in inhibiting the SIM transformation from $\beta$ to $\alpha$ ", thereby giving rise to a slighter SIM transformation in CR specimen compared to that in ST specimen.

Lattice parameters of $\beta$ parent phase and $\alpha^{\prime \prime}$ martensite in CR specimen were extracted from the SXRD 1-D patterns, and their evolution as a function of macroscopic strain is plotted in Fig. $7 b$ and 7c, respectively. It can be seen from Fig. $7 b$ that the parameter of $a_{\beta}$ increases with increasing macroscopic strain to $\sim 3 \%$ due to the elastic deformation of $\beta$ phase and then remains almost constant with a further increase in macroscopic strain up to $5 \%$ owing to the plastic deformation of residual $\beta$. In the case of $\alpha^{\prime \prime}$ martensite (Fig. 7c), it is seen that the parameter of $a_{\alpha "}$ remains about constant during the entire tensile loading, while the $c_{\alpha^{\prime \prime}}$ decreases with macroscopic strain within the strain range of $0-3 \%$, and then stays approximately constant with further loading to $5 \%$. In contrast, the parameter of $b_{\alpha^{\prime \prime}}$ increases with increasing macroscopic strain to $3 \%$ and then keeps approximately constant with further increasing macroscopic strain to $5 \%$. This initial increase of $b_{\alpha^{\prime \prime}}$ is expected to promote the formation of specific $\alpha^{\prime \prime}$ martensite variant, i.e. $\alpha^{\prime \prime}$ variant with $b_{\alpha^{\prime \prime}}$-axis $\left([020]_{\alpha^{\prime \prime}}\right)$ being parallel with tensile direction, to accommodate the external strain to the greatest extent. Indeed, this is verified by the fact that in the loading direction, the $(020)_{\alpha "}$ diffraction peak intensifies with macroscopic strain, accompanied by the decrease and even disappearance of $(002)_{\alpha^{\prime \prime}}($ Fig. 6b). Based on the evolution of lattice parameters in Fig. 7c, a demarcation point between elastic and plastic deformation is confirmed at a strain of $\sim 3 \%$ for $\alpha "$ martensite, nearly identical to that of $\beta$ phase.

According to the above SXRD results of CR specimen, the deformation behaviour of CR Ti-33Nb-4Sn alloy can be clarified straightforwardly and is depicted on the macroscopic stress-strain curve of CR Ti-33Nb-4Sn specimen in Fig. 7d. When 
the macroscopic strains below 3.5\%, the SIM transformation from $\beta$ to $\alpha$ " occurred in a small degree. At the same time, the reorientation of pre-existing $\alpha^{\prime \prime}$ variants also takes place below a strain of $3.5 \%$, as evidenced by the fact that the diffraction intensity of $(002)_{\alpha^{\prime \prime}}$ peak decreases and even disappears with increasing macroscopic strain in the loading direction (Fig. 6b), though the whole integrated intensity of the $(002)_{\alpha "}$ diffraction peak increases within the strain range of $0-3.5 \%$ (Fig. 7a). In addition to SIM transformation and the reorientation of pre-existing $\alpha^{\prime \prime}$ variants, the $\beta$ and $\alpha "$ phases concurrently experience elastic deformation within the macroscopic strain range of $0-3 \%$. At strains in excess of 3\%, the re-oriented and stress-induced $\alpha^{\prime \prime}$ phase and the remaining $\beta$ phase are deformed plastically. Here, an important point to note is that the CR specimen performs nonlinear deformation, instead of a visible "stress plateau" that exists in the ST specimen (Fig. 4d). Previous investigations have shown that the presence of "stress plateau" is mainly caused by the extensive SIM transformation and/or $\alpha^{\prime \prime}$ martensite variants reorientation occurred intensively within a narrow stress/strain range $[18,37,42]$. As show in Fig. $6 \mathrm{~b}$ and $7 \mathrm{a}$, the extent of SIM transformation and $\alpha^{\prime \prime}$ martensite variants reorientation is much smaller in CR Ti-33Nb-4Sn alloy than that in ST specimen. Therefore, the absence of "stress plateau" in $\mathrm{CR}$ Ti-33Nb-4Sn alloy is reasonably attributed to its slighter SIM transformation and less $\alpha^{\prime \prime}$ martensite variants reorientation, compared with that in ST specimen.

\subsection{Deformation behaviour of CRA Ti-33Nb-4Sn alloy}

Upon annealing at $673 \mathrm{~K}$ for 20 minutes, $\alpha$ " martensite formed during quenching and cold rolling is reversibly reverted to $\beta$ parent phase, and most of the $\beta$ phase is retained at room temperature, with a few nano-sized $\alpha$ precipitates. Due to low annealing temperature and short annealing time, this annealing does not result in significant recrystallization, and high density of dislocations as well as grain 
boundaries can still be observed within $\beta$ matrix. A more detailed description of the microstructure of CRA Ti-33Nb-4Sn alloy can be found in a previous literature [29]. Since most of the $\beta$ phase is retained at room temperature and no pre-existing $\alpha^{\prime \prime}$ martensite is observed after annealing, the main objective of the study in this section is to investigate the stability of $\beta$ phase with respect to SIM transformation under uniaxial tensile loading. Here, since the volume fraction of nano-sized $\alpha$ in CRA specimen is quite low (see Fig. S2 in supplementary information), the diffraction intensity of $\alpha$ is not strong enough for a precise peak fitting by in situ SXRD. Additionally, $\alpha$ is generally considered as a stable phase, which cannot be transformed into $\alpha^{\prime \prime}$ martensite during loading, in Ti alloys $[1,14]$. Thus, the deformation behavior of a few nano-sized $\alpha$ precipitates is not considered in the present study.

Fig. 8a shows the evolution of SXRD 1-D patterns (within the $d$-spacing range of

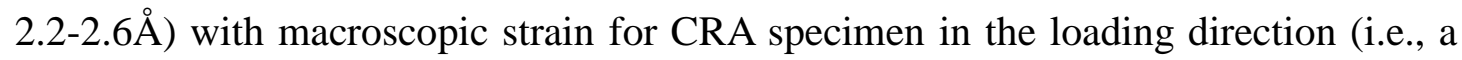
$10^{\circ}$ azimuthal bin around $90^{\circ}$ and $270^{\circ}$ ). Within this $d$-spacing range, only $\{110\}_{\beta}$ diffraction peak is observed, without the presence of any $\alpha$ " martensite peaks, during the whole tensile loading. The $d$-spacing of $\{110\}_{\beta}$ first increases with increasing macroscopic strain to $\sim 3 \%$, and then remains almost constant with a further increase in strain to $5 \%$. The evolution of SXRD 1-D patterns (within the $d$-spacing range of 2.2-2.6 $\AA$ ) with macroscopic strain in the Poisson direction (i.e., a $10^{\circ}$ azimuthal bin around $0^{\circ}$ and $180^{\circ}$ ) is shown in Fig. 8b. In the Poisson direction, the $d$-spacing of $\{110\}_{\beta}$ first decreases with increasing macroscopic strain to $\sim 3 \%$, and then stays almost constant with further increasing macroscopic strain to $5 \%$. The decrease of the $d$-spacing of $\{110\}_{\beta}$ is reasonably attributed to a compressive stress in the Poisson direction when a uniaxial tensile stress is applied along the axial direction. Again, no visible $\alpha^{\prime \prime}$ martensite diffraction peaks from $(002)_{\alpha^{\prime \prime}}$ and $(020)_{\alpha^{\prime \prime}}$ are observed during 
the whole tensile loading. According to the SXRD results shown in Fig. 8a and 8b, it can be concluded that the stability of $\beta$ phase in the CRA specimen is high enough to survive against SIM transformation from $\beta$ to $\alpha$ ".

In order to further clarify the response of $\beta$ phase to uniaxial tensile, the evolution of the lattice parameter of $\beta\left(a_{\beta}\right)$ during loading is extracted and shown in Fig. 8c. One can see that the parameter of $a_{\beta}$ first increases with macroscopic strain up to a strain of $\sim 3 \%$, and then remains approximately unchanged with a further increase in macroscopic strain. Clearly, the initial increase of $a_{\beta}$ is attributed to elastic deformation of $\beta$, whereas the constant of $a_{\beta}$, irrespective of further increasing macroscopic strain, corresponds to the plastic deformation of $\beta$. According to the results shown above, a demarcation point, at a strain of about $3 \%$, between elastic and plastic deformation can be determined straightforwardly for CRA Ti-33Nb-4Sn alloy, and the regions of different deformation mechanisms of CRA Ti-33Nb-4Sn alloy is depicted on the macroscopic stress-strain curve in Fig. 8d. Based on the results shown in Fig. $8 \mathrm{c}$ and $8 \mathrm{~d}$, one can see that a value of $\sim 2.9 \%$ for microscopic elastic lattice strain and a value of $\sim 3.0 \%$ for macroscopic elastic strain can be obtained in CRA Ti-33Nb-4Sn alloy, indicating that upon appropriate thermo-mechanical treatment (i.e., cold rolling plus annealing), the Ti-33Nb-4Sn alloy can exhibit a huge elastic deformability.

The level of this elastic deformability is the hugest ever recorded for a solid and bulk titanium alloy, and is much huger than those of the currently available metallic materials for elastic applications $[1,2,7,15,43]$. This huge elastic deformability might be related to the moderate $\beta$-phase stability of CRA Ti-33Nb-4Sn alloy, which is high enough to survive against SIM transformation from $\beta$ to $\alpha^{\prime \prime}$ but still much lower than that of most of the currently available $\beta$-type alloys due to its low $\beta$-phase stability 
with respect to $\{110\}<1 \overline{10}>$ shear (i.e., $C^{\prime}$ ) and $\{001\}<100>$ shear (i.e., $C_{44}$ ) [30]. Our experimental results show that with the assistance of high density of dislocations and grain boundaries, the $\beta$ phase, which contains the low content of $\beta$ stabilizers and has low stability of $\beta$-phase, can survive against SIM transformation and perform a huge elastic deformability. We believe that these results can shed light on design and development of novel $\beta$-type titanium alloys with large elastic limit.

\section{Conclusions}

In situ synchrotron X-ray diffraction study was carried out on a recently developed Ti-33Nb-4Sn (wt. \%) alloy during uniaxial tensile loading, with special attention is focused on the relationship between SIM transformation and deformation behaviour under different thermo-mechanical treatment conditions. The main conclusions obtained are summarized as follows:

(1) Though the SIM transformation covers a wide strain range of $0.5-14 \%$ in ST alloy, some remaining $\beta$ parent phase can still be observed at a strain of $14 \%$, indicating that the SIM transformation is incomplete in ST alloy. This SIM transformation exacerbates the texture of the parent $\beta$ and $\alpha^{\prime \prime}$ martensite, allowing a precise and direct measurement of the crystallographic orientation relationship between $\beta$ and $\alpha$ " phases.

(2) During the uniaxial tensile loading of ST alloy, the parameter of $b_{\alpha^{\prime \prime}}\left([020]_{\alpha^{\prime \prime}}\right)$ increases with macroscopic strain within strain range of $1.5-4.7 \%$, resulting in that the $\alpha^{\prime \prime}$ variants, with $b_{\alpha^{\prime \prime}}$ axis $\left([020]_{\alpha^{\prime \prime}}\right)$ being parallel to tensile direction, are formed preferentially to accommodate the macroscopic strain in tensile loading. This offers a reasonable explanation for why the $(020)_{\alpha "}$ peak intensifies, but the $\{110\}_{\beta}$ and $(002)_{\alpha^{\prime \prime}}$ peaks reduce and even eliminate along the loading direction during uniaxial loading.

(3) Similar SIM transformation behaviour also takes place in CR alloy with the exception that the extent of SIM transformation and pre-existing $\alpha^{\prime \prime}$ variants 
reorientation in CR specimen is much slighter than that in ST specimen. This slighter SIM transformation leads to "nonlinear deformation" in CR alloy, which is different from visible "stress plateau" that exists in ST specimen.

(4) Upon annealing at $673 \mathrm{~K}$ for 20 minutes, the $\beta$ phase survives against SIM transformation from $\beta$ to $\alpha^{\prime \prime}$ due to the suppression effects of dislocations and grain boundaries on SIM transformation. In this case, the Ti-33Nb-4Sn alloy can exhibit a huge elastic deformability ( $2.9 \%$ for microscopic elastic lattice strain and $\sim 3.0 \%$ for macroscopic elastic strain) owing to its low $\beta$-phase stability with respect to $\{110\}<1 \overline{1}$ 0> shear (i.e., $C^{\prime}$ ) and $\{001\}<100>$ shear (i.e., $\mathrm{C}_{44}$ ).

(5) The activation sequence of different deformation mechanisms and the regions of different deformation mechanisms, involving elastic and plastic deformation of $\beta$ phase, elastic deformation of the pre-existing $\alpha^{\prime \prime}$, re-oriented and stress-induced $\alpha^{\prime \prime}$ variants, reorientation of the pre-existing $\alpha^{\prime \prime}$, SIM transformation, and plastic deformation of the re-oriented and stress-induced $\alpha^{\prime \prime}$ martensite, in Ti-33Nb-4Sn alloys under different thermo-mechanical treatment conditions, can be clarified unambiguously based on the experimental results from tensile tests and SXRD.

\section{Acknowledgments}

The authors greatly appreciate the financial support from the National Natural Science Foundation of China (51401088, 51471017, 51601069, 51601217), the Special Financial Grant from the China Postdoctoral Science Foundation (2016T90424) and the Natural Science Foundation of Jiangsu Province (BK20140549, BK20160514, BK20160255). The use of the Advanced Photon Source was supported by the US Department of Energy, Office of Science, and Office of Basic Energy Science, under Contract No. DE-AC02-06CH11357. 


\section{References}

[1] A. Biesiekierski, J. Lin, Y. Li, D. Ping, Y. Yamabe-Mitarai, C. Wen, Acta Biomater. 32 (2016) 336-347.

[2] P.S. Nnamchi, C.S. Obayi, I. Todd, M.W. Rainforth, J. Mech. Behav. Biomed. Mater. 60 (2016) 68-77.

[3] X. Zhao, M. Niinomi, M. Nakai, G. Miyamoto, T. Furuhara, Acta Biomater. 7 (2011) 3230-3236.

[4] M. Niinomi, T. Akahori, N. Masaaki, Mater. Sci. Eng. C 28 (2008) 406-413.

[5] D.R. Sumner, T.M. Turner, R. Igloria, R.M. Urban, J.O. Galante, J. Biomech. 31 (1998) 909-917.

[6] E. Eisenbarth, D. Velten, M. Muller, R. Thull, J. Breme, Biomaterials 25 (2004) 5705-5713.

[7] S.X. Liang, X.J. Feng, L.X. Yin, X.Y. Liu, M.Z. Ma, R.P. Liu, Mater. Sci. Eng. C 61 (2016) 338-343.

[8] D. Kuroda, M. Niinomi, M. Morinaga, Y. Kato, T. Yashiro, Mater. Sci. Eng. A 243 (1998) 244-249.

[9] M.J. Long, H.J. Rack, Biomaterials 19 (1998) 1621-1639.

[10] M. Tane, S. Akita, T. Nakano, K. Hagihara, Y. Umakoshi, M. Niinomi, H. Nakajima, Acta Mater. 56 (2008) 2856-2863.

[11] L.M. Elias, S.G. Schneider, S. Schneider, H.M. Silva, F. Malvisi, Mater. Sci. Eng. A 43 (2006) 108-112.

[12] M. Abdel-Hady, K. Hinoshita, M. Morinaga, Scr. Mater. 55 (2006) 477-480.

[13] Y. Tian, Z. Yu, C.Y.A. Ong, W. Cui, Mater. Lett. 145 (2015) 283-286.

[14] S. Guo, Q.K. Meng, X.N. Cheng, X.Q. Zhao, Mater. Lett. 133 (2014) 236-239.

[15] Y.L. Zhou, D.M. Luo, Mater. Charact. 62 (2011) 931-937.

[16] Y.L. Hao, S.J. Li, S.Y. Sun, C.Y. Zheng, R. Yang, Acta Biomater. 3 (2007) 277-286.

[17] J.P. Liu, Y.D. Wang, Y.L. Hao, H.L. Wang, Y. Wang, Z.H. Nie, R. Su, D. Wang, Y. Ren, Z.P. Lu, J.G. Wang, X.D. Hui, R. Yang, Acta Mater. 81 (2014) 476-486.

[18] Y. Al-Zain, H.Y. Kim, H. Hosoda, T.H. Nam, S. Miyazaki, Acta Mater. 58 (2010) 4212-4223.

[19] D.C. Zhang, Y.F. Mao, M. Yan, J.J. Li, E.L. Su, Y.L. Li, S.W. Tan, J.G. Lin, J. Mech. Behav. Biomed. Mater. 20 (2013) 29-35.

[20] P.J.S. Buenconsejo, H.Y. Kim, S. Miyazaki, Scr. Mater. 64 (2011) 1114-1117.

[21] J. Fu, A. Yamamoto, H.Y. Kim, H. Hosoda, S. Miyazaki, Acta Biomater. 17 (2015) 56-67.

[22] E. Bertrand, P. Castany, Y. Yang, E. Menou, T. Gloriant, Acta Mater. 105 (2016) 94-103.

[23] Y.W. Chai, H.Y. Kim, H. Hosoda, S. Miyazaki, Acta Mater. 57 (2009) 4054-4064. [24] S.Q. Wu, D.H. Ping, Y. Yamabe-Mitarai, T. Kitashima, G.P. Li, R. Yang, J. Alloys 
Compd. 577S (2013) S423-S426.

[25] F. Sun, Y.L. Hao, S. Nowak, T. Gloriant, P. Laheurte, F. Prima, J. Mech. Behav. Biomed. Mater. 4 (2011) 1864-1872.

[26] S. Guo, Z.Z. Bao, Q.K. Meng, L. Hu, X.Q. Zhao, Metall. Mater. Trans. A 43 (2012) 3447-3451.

[27] S. Hanada, N. Masahashi, T.K. Jung, Mater. Sci. Eng. A 588 (2013) 403-410.

[28] H. Matsumoto, S. Watanabe, S. Hanada, J. Alloys Compd. 439 (2007) 146-155.

[29] S. Guo, Q.K. Meng, Q.M. Wei, X.Q. Zhao, H.B. Xu, Sci. Rep. 5 (2015) 514688.

[30] Y.P. Hou, S. Guo, X.L. Qiao, T. Tian, Q.K. Meng, X.N. Cheng, X.Q. Zhao, J. Mech. Behav. Biomed. Mater. 59 (2016) 220-225.

[31] Y. Yang, P. Castany, M. Cornen, F. Prima, S.J. Li, Y.L. Hao, T. Gloriant, Acta Mater. 88 (2015) 25-33.

[32] P. Castany, A. Ramarolahy, F. Prima, P. Laheurte, C. Curfs, T. Gloriant, Acta Mater. 88 (2015) 102-111.

[33] V.A. Vorontsov, N.G. Jones, K.M. Rahman, D. Dye, Acta Mater. 88 (2015) 323-333.

[34] S.L. Raghunathan, M.A. Azeem, D. Collins, D. Dye, Scr. Mater. 59 (2008) 1059-1062.

[35] S. Cai, M.R. Daymond, Y. Ren, J.E. Schaffer, Acta Mater. 59 (2011) 1464-1473.

[36] K. Otsuka, X.B. Ren, Prog. Mater. Sci. 50 (2005) 511-678.

[37] H.Y. Kim, Y. Ikehara, J.I. Kim, H. Hosoda, S. Miyazaki, Acta Mater. 54 (2006) 2419-2429.

[38] S.J. Li, Y.W. Zhang, B.B. Sun, Y.L. Hao, R. Yang, Mater. Sci. Eng. A 480 (2008) 101-108.

[39] S. Guo, Q.K. Meng, L. Hu, G.Y. Liao, X.Q. Zhao, H.B. Xu, J. Alloys Compd. 550 (2013) 35-38.

[40] Y.L. Hao, M. Niinomi, D. Kuroda, K. Fukunaga, Y.L. Zhou, R. Yang, A. Suzuki, Metall. Mater. Trans. A 33 (2002) 3137-3144.

[41] H. Matsumoto, S. Watanabe, S. Hanada, Mater. Trans. 46 (2005) 1070-1078.

[42] S. Nobuhito, N. Mitsuo, A. Toshikazu, T. Junji, T. Hiroyuki, Mater. Sci. Eng. C 25 (2005) 363-369.

[43] A. Ghaei, D.E. Green, A. Aryanpour, Mater. Des. 88 (2015) 461-470.

\section{Figure captions:}

Figure 1. Initial SXRD 1-D pattern (a) and convention XRD profile (b) of ST Ti-33Nb-4Sn alloy before loading (i.e., at a macroscopic strain of 0\%). The inset in (a) is an enlarged SXRD pattern within the $d$-spacing range of $1.25-1.45 \AA$, showing clearly the presence of $\alpha^{\prime \prime}$ martensite. Note that no visible diffraction peak from 
$(002)_{\alpha^{\prime \prime}}$ is detected in figure (b), while this $(002)_{\alpha^{\prime \prime}}$ peak can be detected clearly alongside $\{110\}_{\beta}$ by SXRD technique in figure (a).

Figure 2. Part of the straightened Debye-Scherrer diffraction rings of ST specimen as a function of macroscopic strain (a), showing a progressive SIM transformation occurred during loading. Original 2-D Debye-Scherrer diffraction rings of ST specimen acquired at $14 \%$ strain (b), where the lattice correspondence between the $\beta$ parent phase and $\alpha$ " martensite can be observed directly.

Figure 3. Evolution of SXRD 1-D patterns (within the $d$-spacing range of 2.2-2.6 ) with macroscopic strain for ST specimen in the loading direction (a) and in the Poisson direction (b). Blue circled symbols label the $\{110\}_{\beta}$ diffraction peaks, black diamond symbols label the $(002)_{\alpha^{\prime \prime}}$ peaks, red squared symbols label the $(020)_{\alpha^{\prime \prime}}$ peaks. The inset in (b) is an enlarged SXRD patterns at strains of $0 \%, 0.5 \%, 1.0 \%, 1.5 \%$ and $2.1 \%$, showing the decrease and even disappearance of $(020)_{\alpha^{\prime \prime}}$ in the Poisson direction.

Figure 4. Evolution of the relative integrated intensity of $\{110\}_{\beta},(002)_{\alpha^{\prime \prime}}$ and $(020)_{\alpha^{\prime \prime}}$ peaks (a), and the parameters of $\beta$ (b) and $\alpha^{\prime \prime}$ (c) phases of ST Ti-33Nb-4Sn specimen during loading. Domains of occurrence of different deformation mechanisms are depicted on macroscopic stress-strain curve of ST Ti-33Nb-4Sn (d).

Figure 5. Bright-field TEM images of CR (a) and ST (b) Ti-33Nb-4Sn specimens, the corresponding selected area electron diffraction (SAD) patterns are shown in their top right corners, respectively.

Figure 6. Part of the straightened Debye-Scherrer diffraction rings of CR Ti-33Nb-4Sn specimen at different strain levels during uniaxial loading (a). Evolution

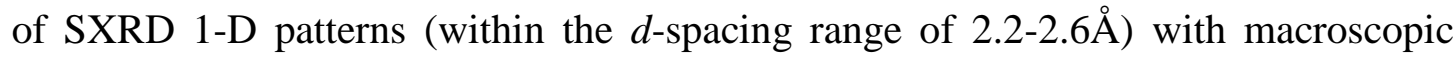
strain for CR Ti-33Nb-4Sn specimen in the loading direction (b). 
Figure 7. Evolution of the relative integrated intensity of $\{110\}_{\beta},(002)_{\alpha^{\prime \prime}}$ and $(020)_{\alpha^{\prime \prime}}$ peaks (a), and the parameters of $\beta$ (b) and $\alpha^{\prime \prime}$ (c) phases of CR Ti-33Nb-4Sn specimen during loading. Domains of occurrence of different deformation mechanisms are depicted on macroscopic stress-strain curve of CR Ti-33Nb-4Sn (d).

Figure 8. Evolution of SXRD 1-D patterns (within the $d$-spacing range of 2.2-2.6 ) with macroscopic strain for CRA Ti-33Nb-4Sn specimen in the loading direction (a) and in the Poisson direction (b). Evolution of the parameters of $\beta$ of CRA Ti-33Nb-4Sn specimen during loading (c). Domains of occurrence of different deformation mechanisms are depicted on macroscopic stress-strain curve of CRA Ti-33Nb-4Sn (d). 

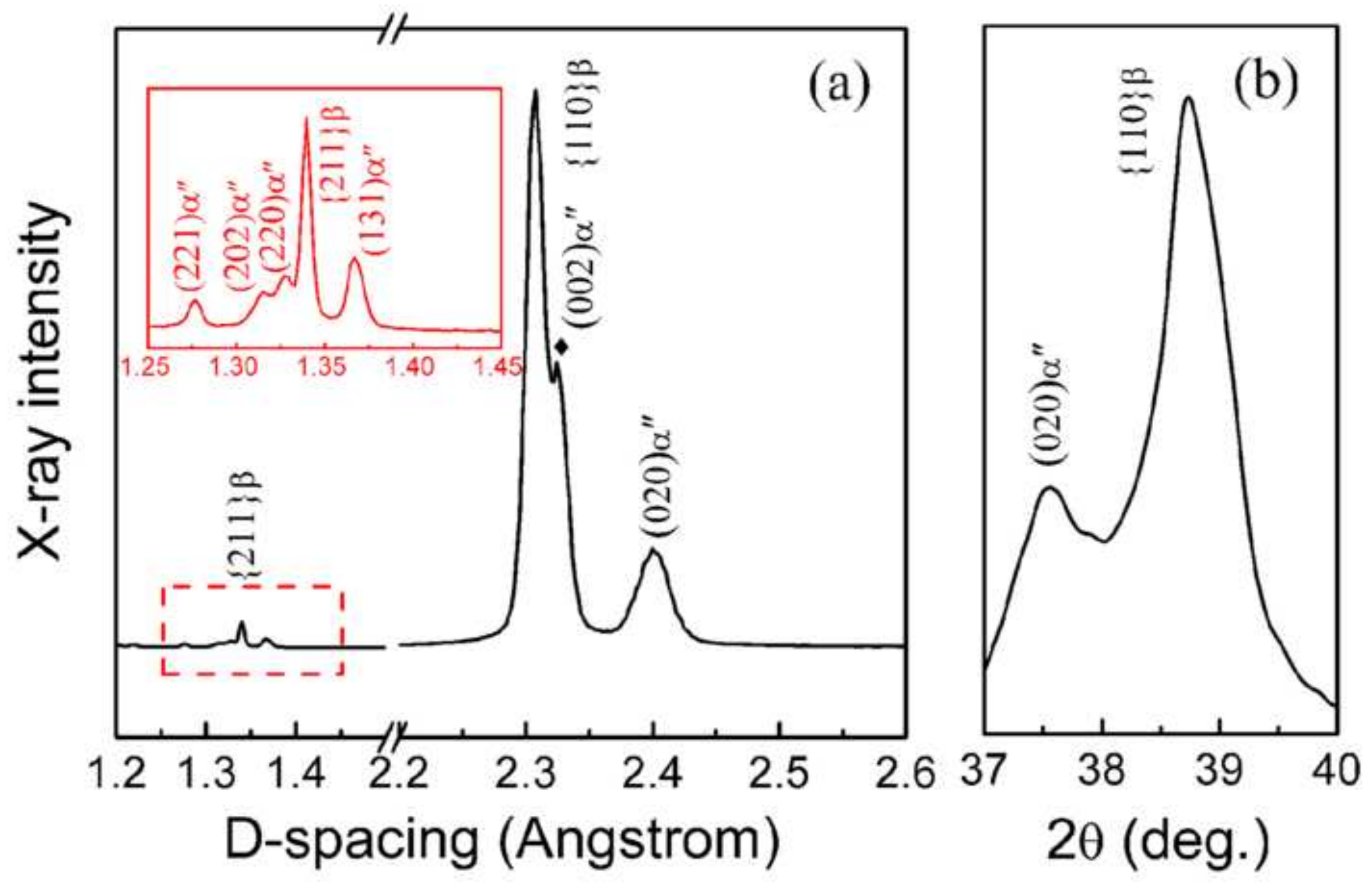


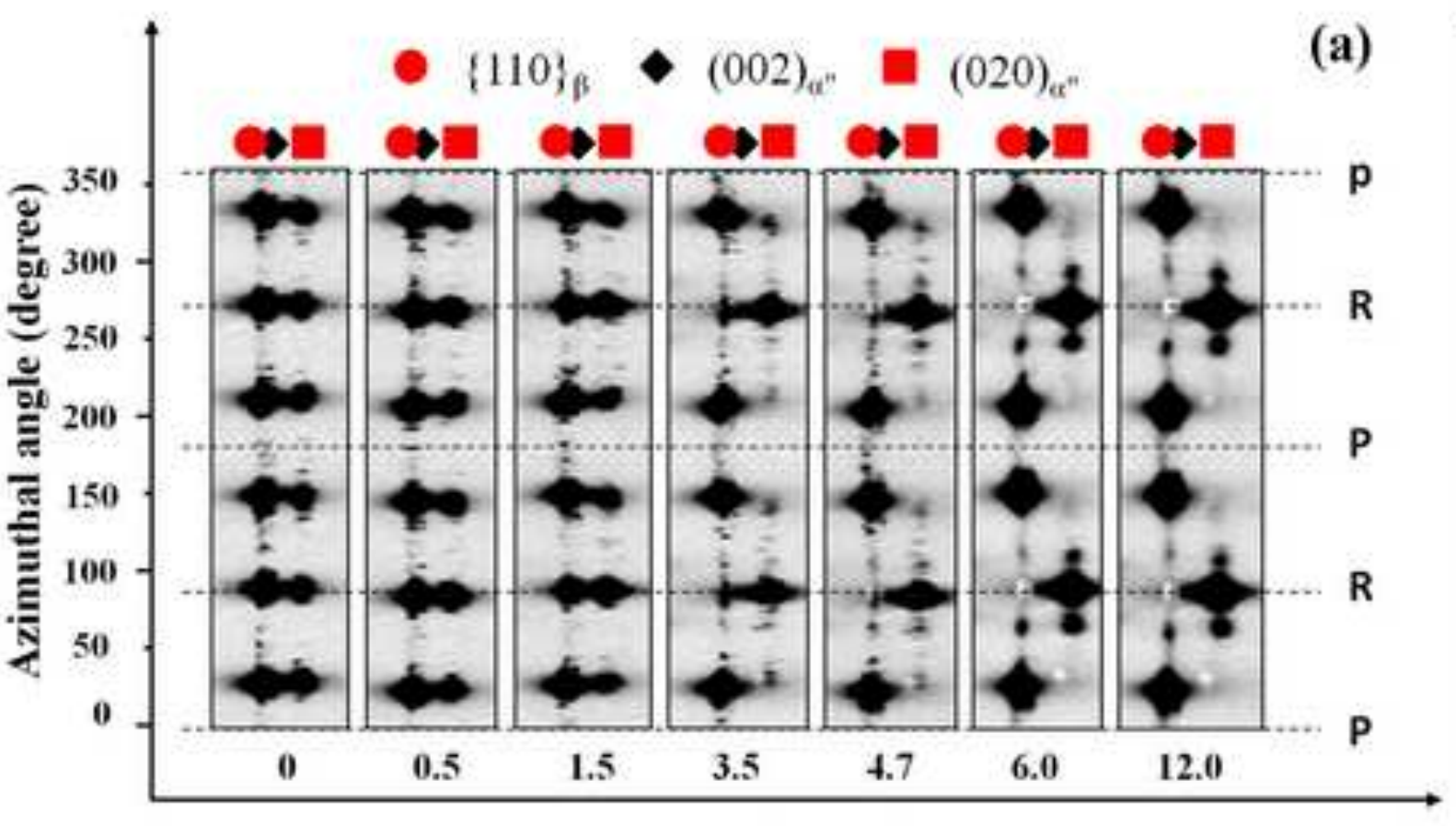

Macroscopic strain (\%)

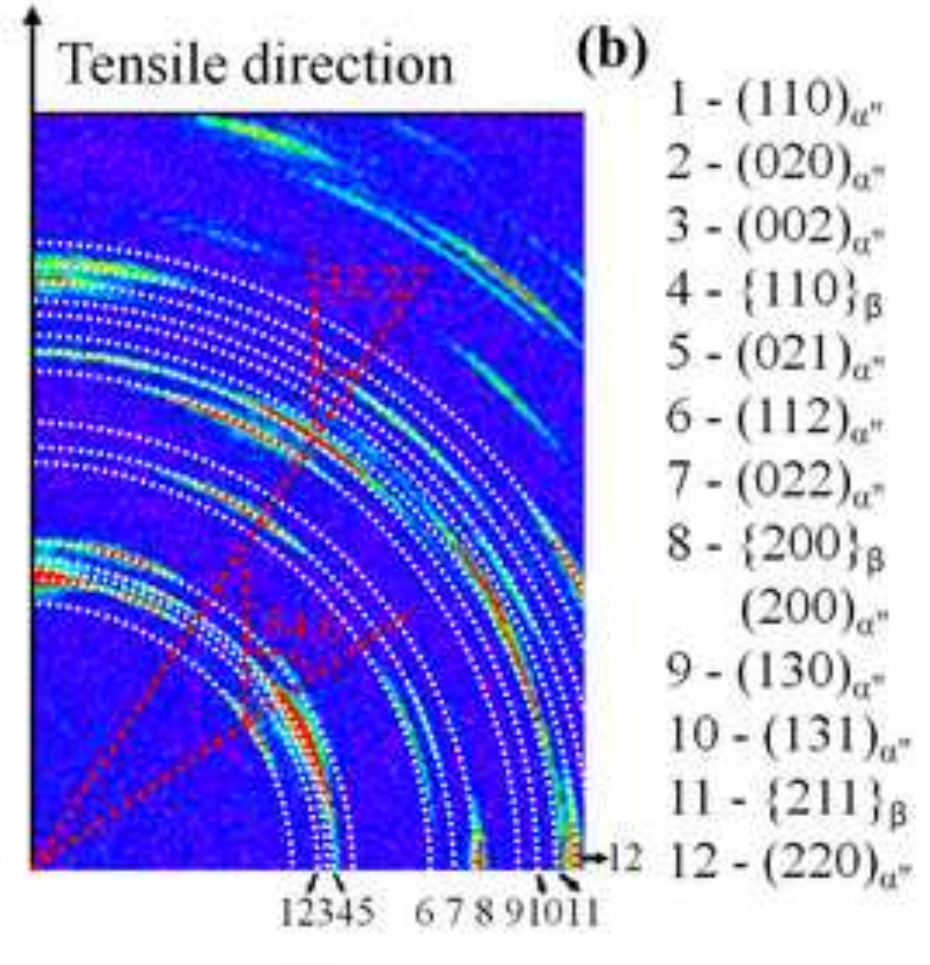



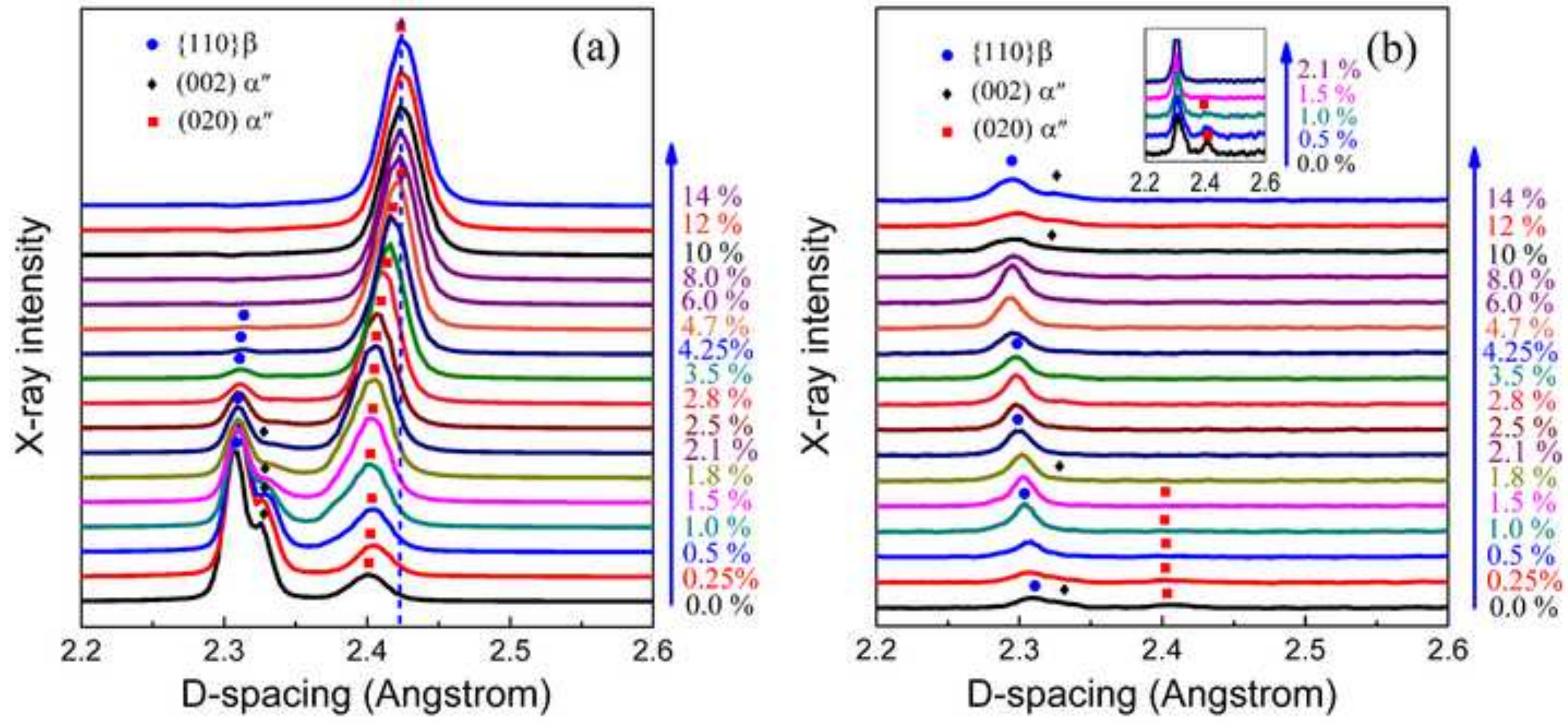

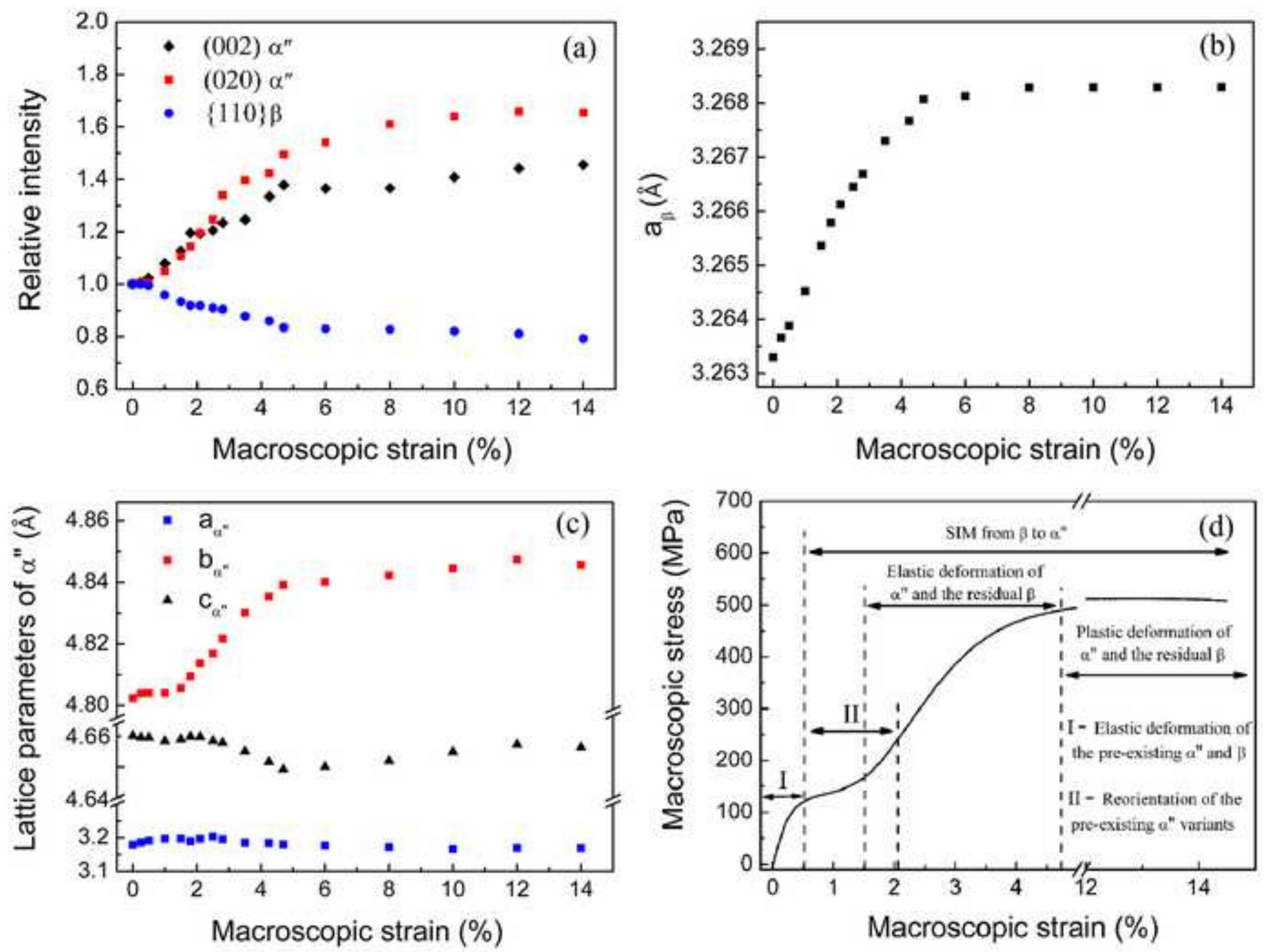

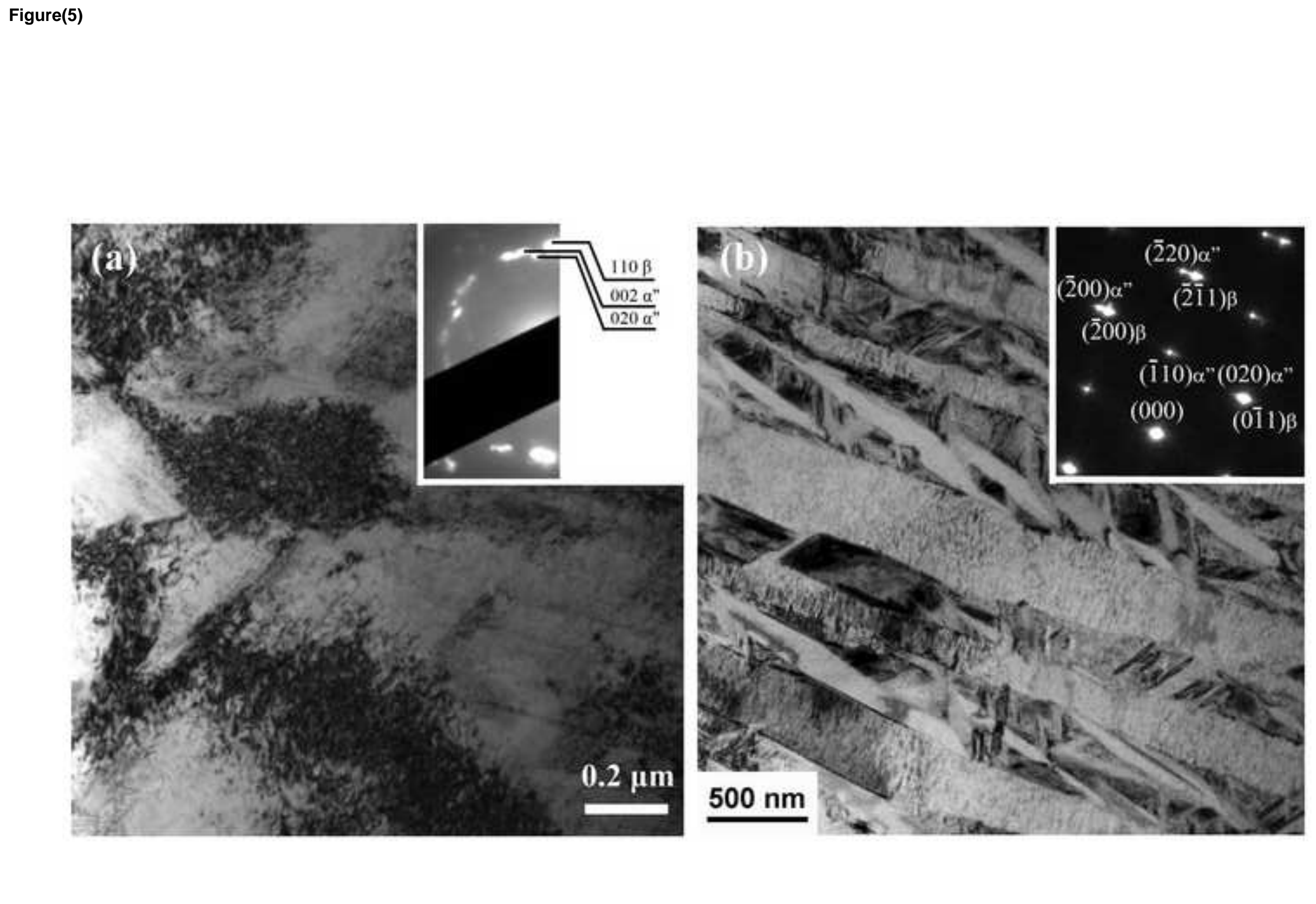

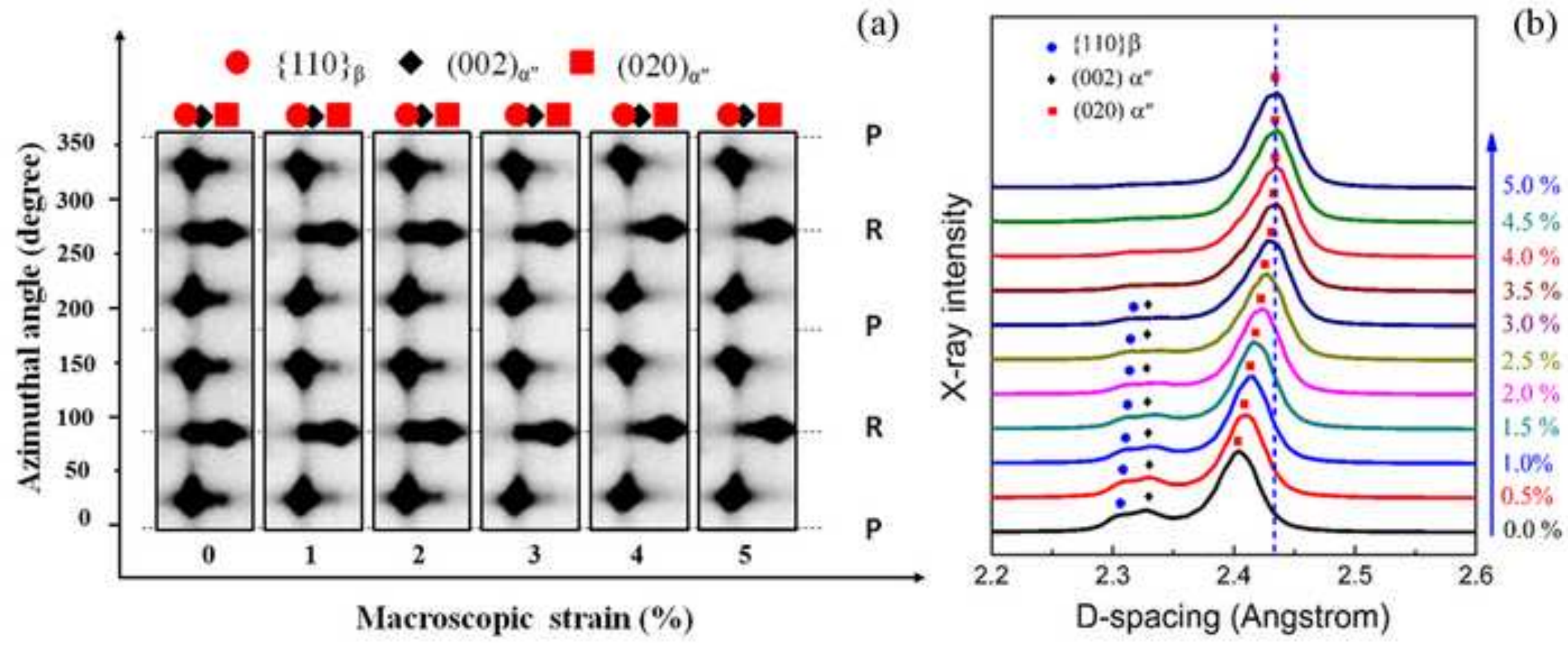

(b)

$.0 \%$
$5 \%$

$.0 \%$

$3.5 \%$

$2.5 \%$

$0 \%$

D-spacing (Angstrom) 

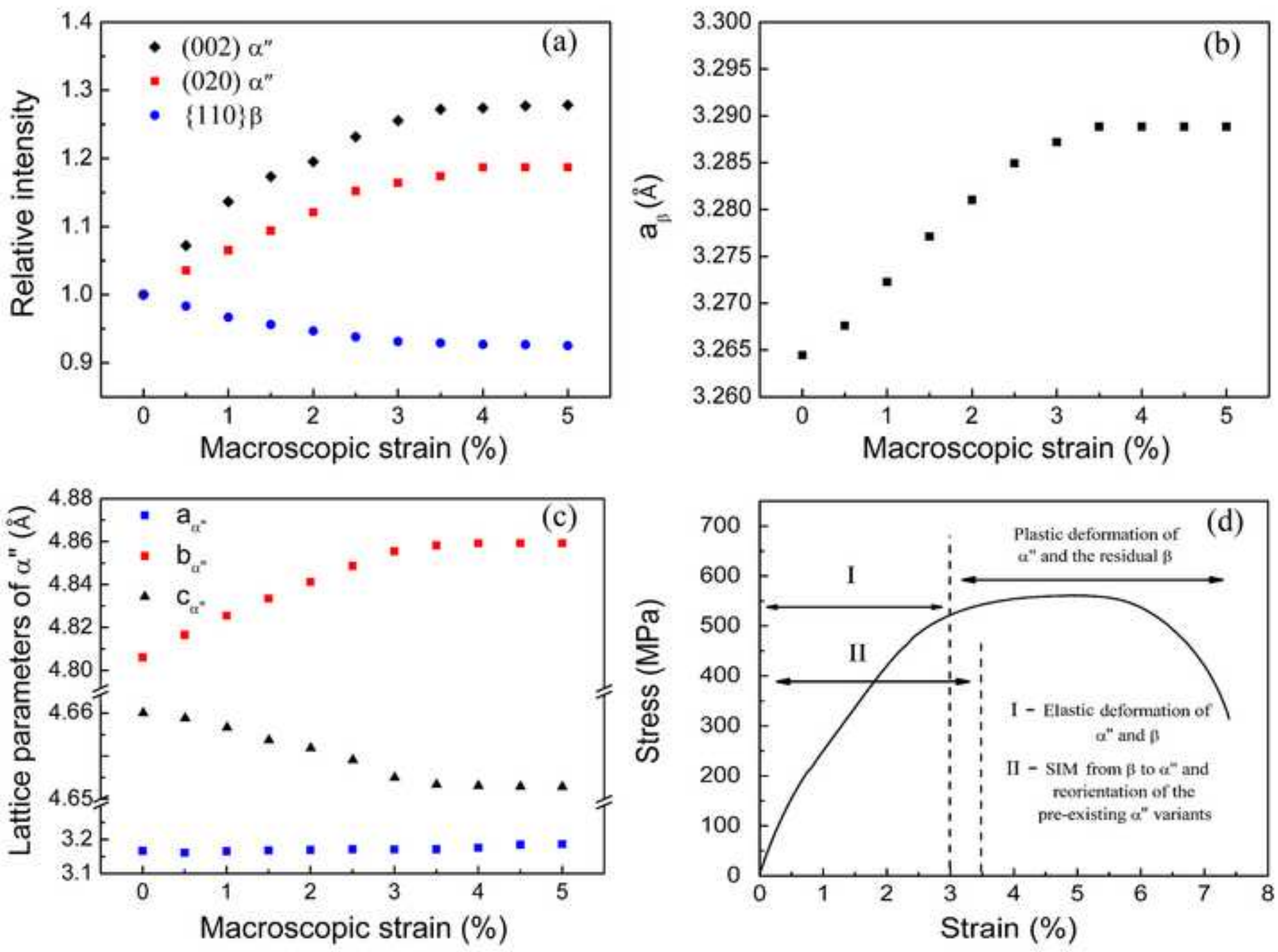


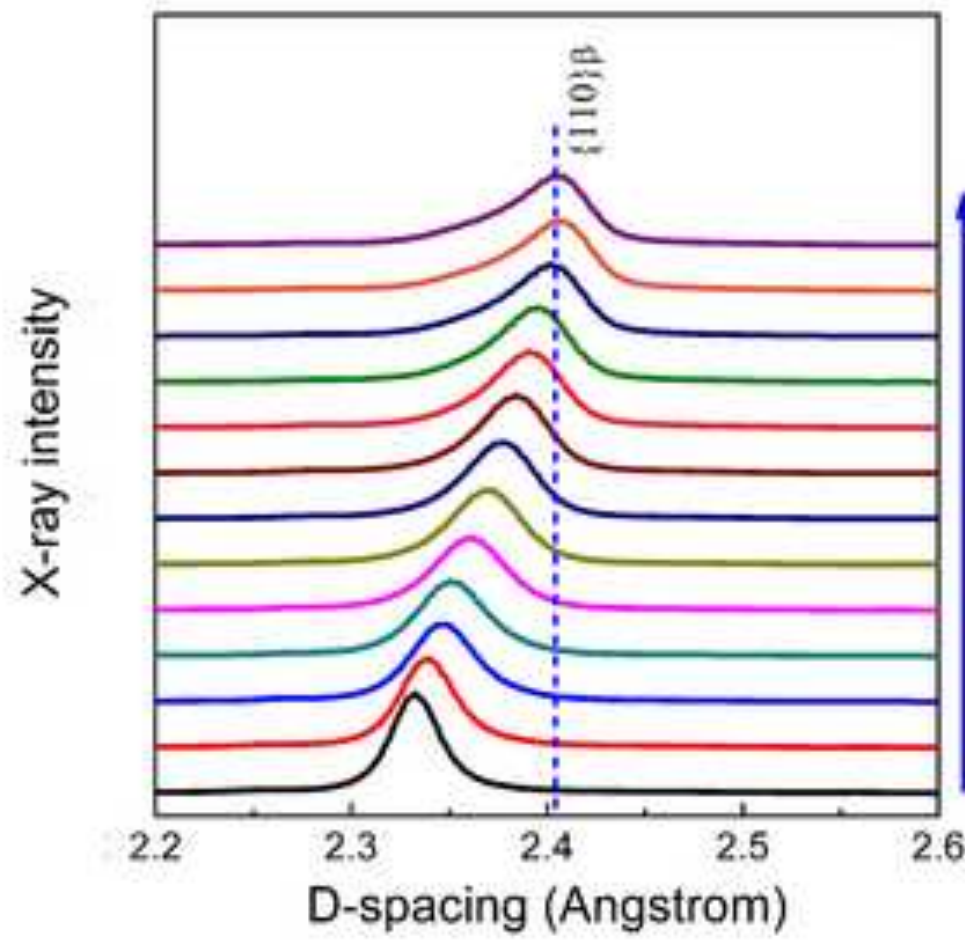

(a)

$5.0 \%$

$4.0 \%$

$3.0 \%$

$2.5 \%$

$2.25 \%$

$2.0 \%$

$1.75 \%$

$1.5 \%$

$1.25 \%$

$1.0 \%$

$0.75 \%$

$0.25 \%$

产

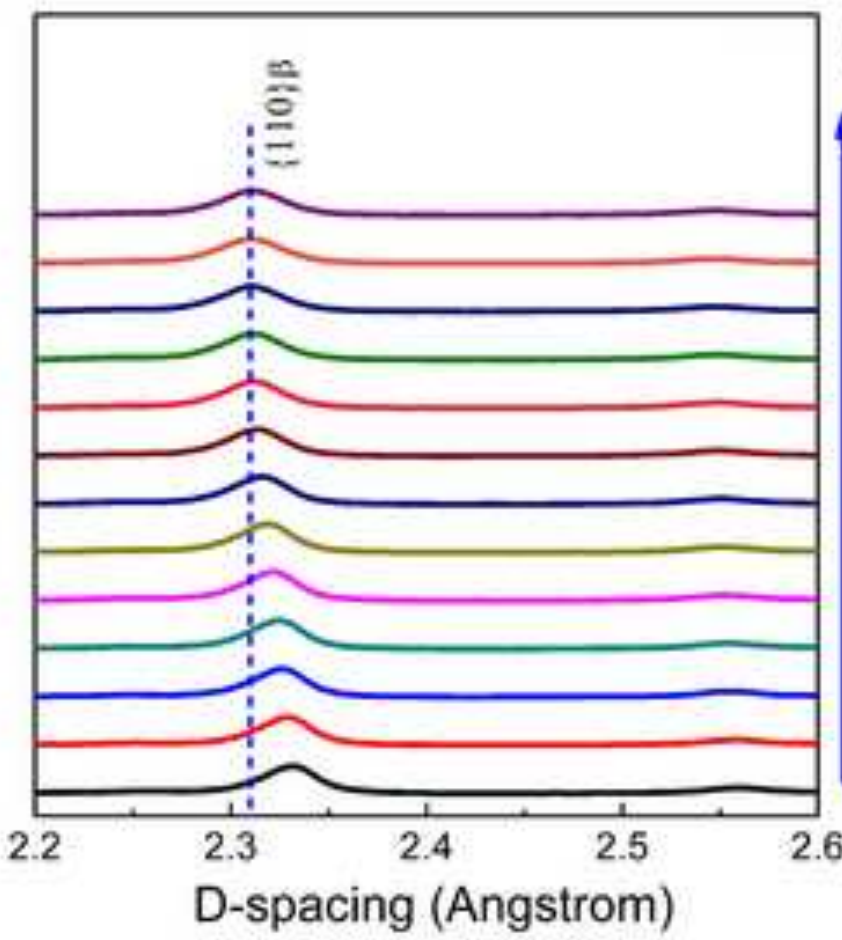

(b)
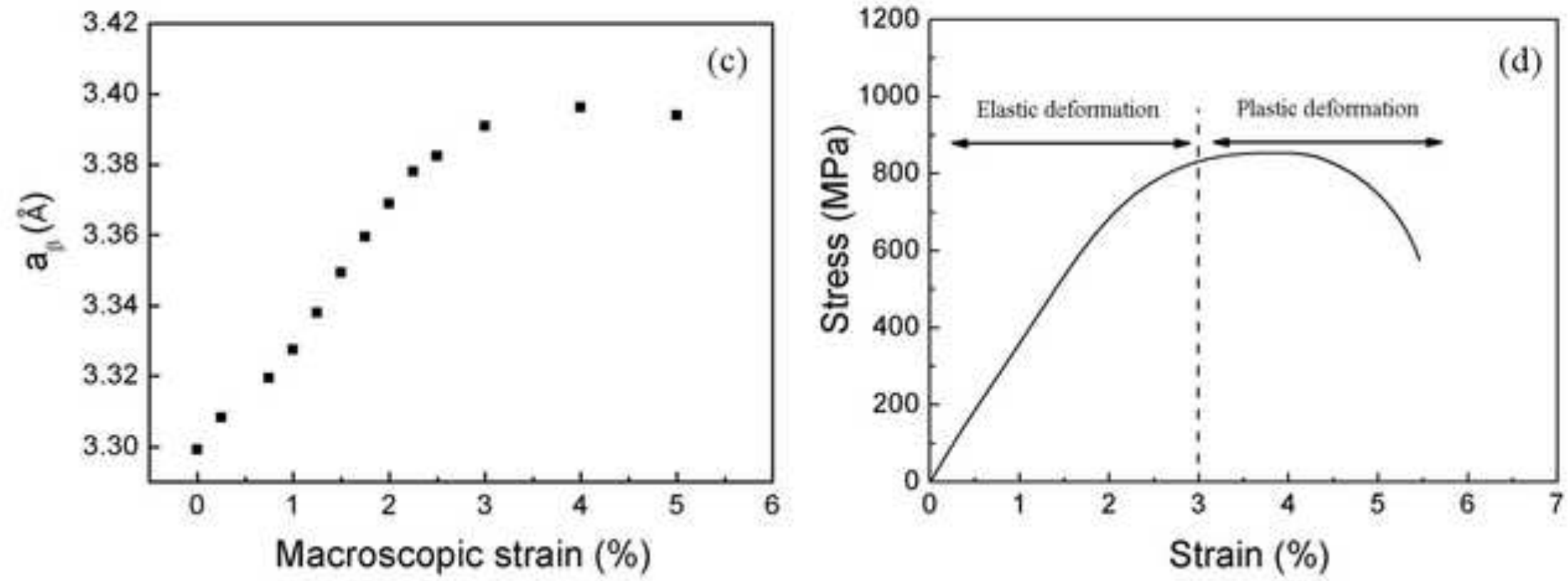